\title{
A atuação neuroendócrina no controle da fome e saciedade e sua relação com a
}

\section{obesidade}

The neuroendocrine role in the control of hunger and satiety and its relationship with obesity El papel neuroendocrino en el control del hambre y la saciedad y su relación con la obesidad

Saray Sallin da Silva

ORCID: https://orcid.org/0000-0002-8889-7830 Instituto Tocantinense Presidente Antônio Carlos, Brasil

E-mail: saray.sallin@gmail.com

Shara Hozana Silva

ORCID: https://orcid.org/0000-0002-9448-2439 Instituto Tocantinense Presidente Antônio Carlos, Brasil

E-mail: shara.hozana@gmail.com

Géssica Adorno Aguiar

ORCID: https://orcid.org/0000-0002-1770-267X Instituto Tocantinense Presidente Antônio Carlos, Brasil E-mail: gessica2198@gmail.com

Stefane Oliveira Batista

ORCID: https://orcid.org/0000-0003-2404-5046 Instituto Tocantinense Presidente Antônio Carlos, Brasil

E-mail: stefanelirio@gmail.com

Ana Clara Marinho Santos

ORCID: https://orcid.org/0000-0002-3219-4976 Instituto Tocantinense Presidente Antônio Carlos, Brasil E-mail: anaclara-ms@hotmail.com

Fernanda Láuria Chaves Bandeira

ORCID: https://orcid.org/0000-0002-2377-870X Instituto Tocantinense Presidente Antônio Carlos, Brasil

E-mail: ferlauriacb@gmail.com

Maryana Teixeira Alves

ORCID: https://orcid.org/0000-0003-1852-3865 Instituto Tocantinense Presidente Antônio Carlos, Brasil E-mail: maryanateixeira09@gmail.com

Áthila Gabriele Ferreira da Silva

ORCID: https://orcid.org/0000-0001-9543-3826

Universidade Federal do Maranhão, Brasil

E-mail: athila.gabriele@ discente.ufma.br

Vanessa Alves de Sousa

ORCID: https://orcid.org/0000-0001-8063-3200 Universidade Federal do Maranhão, Brasil E-mail: vanessa.as@discente.ufma.br

Cicera Natália da Silva Rodrigues

ORCID: https://orcid.org/0000-0002-9034-1989

Universidade Federal do Maranhão, Brasil E-mail: cicera.natalia@discente.ufma.br

Nibsyan Cristina da Silva

ORCID: https://orcid.org/0000-0002-0549-0366 Instituto Tocantinense Presidente Antônio Carlos, Brasil E-mail: nibsyan@ hotmail.com

Letícia Aparecida de Souza

ORCID: https://orcid.org/0000-0001-8571-8123 Instituto Tocantinense Presidente Antônio Carlos, Brasil E-mail: leticia.biofarmed@gmail.com

Thaysa Renata Jorge Oliveira

ORCID: https://orcid.org/0000-0003-0829-793X Instituto Tocantinense Presidente Antônio Carlos, Brasil E-mail: thaysarenata22@hotmail.com

Bianca Silva Cardoso

ORCID: https://orcid.org/0000-0002-2129-4023

Universidade de Rio Verde, Brasil E-mail: bianca.cardoso.vest@gmail.com 


\author{
Josyane Borges da Silva Gonçalves \\ ORCID: https://orcid.org/0000-0002-1420-8879 \\ Instituto Tocantinense Presidente Antônio Carlos, Brasil \\ E-mail: josyaneborgess@gmail.com
}

\begin{abstract}
Resumo
A obesidade é um distúrbio crônico de origem multifatorial (fatores neuroendócrinos, psíquicos, intestinais e genéticos) que resulta de um desequilíbrio metabólico-energético, pelo qual um excesso de longo prazo da ingestão de energia sobre o gasto, leva ao armazenamento em forma de gordura. O controle do balanço energético é realizado pelo sistema nervoso central por meio de conexões neuroendócrinas, em que hormônios periféricos circulantes, como a leptina e a insulina, sinalizam neurônios especializados do hipotálamo sobre os estoques de gordura do organismo e induzem respostas apropriadas para a manutenção da estabilidade desses estoques. Assim, o balanço energético positivo por longo período, determinado pela ingestão de macronutrientes, pelo gasto energético e pela termogênese, resultará em ganho de peso corporal na forma de gordura, enquanto o balanço energético negativo resultará no efeito oposto. Nesta revisão sistemática de trabalhos publicados entre 2008 e 2021, serão discutidos os mecanismos neuroendócrinos que atuam no controle da fome e saciedade e como eles implicam na obesidade. Evidenciando assim, que essa regulação é realizada por um mecanismo complexo que precisa ser muito bem compreendido para que se possa tratar ou até mesmo prevenir a obesidade.
\end{abstract}

Palavras-chave: Obesidade; Fome; Saciedade.

\begin{abstract}
Obesity is a chronic disorder of multifactorial origin (neuroendocrine, psychic, intestinal and genetic factors) that results from a metabolic-energetic imbalance, whereby a long-term excess of energy intake over expenditure leads to accumulation in the form of fat. The control of the energy balance is carried out by the central nervous system by means of necessary neuroendocrines, in which peripheral circulating hormones, such as leptin and insulin, signal specialized neurons of the hypothalamus over the body's fat stores and induce adequate responses for the maintenance of stocks god stability. Thus, the positive energy balance for a long period, determined by macronutrient intake, energy expenditure and thermogenesis, will result in body weight gain in the form of fat, while the negative energy balance will result in no opposite effect. In this systematic review of works published between 2008 and 2021 , neuroendocrine mechanisms that control hunger and satiety, and their relationship with obesity, will be discussed. Thus evidencing that this regulation is carried out by a complex mechanism that needs to be very well understood in order to treat or even prevent obesity.
\end{abstract}

Keywords: Obesity; Hunger; Satiety.

\title{
Resumen
}

La obesidad es un trastorno crónico de origen multifactorial (factores neuroendocrinos, psíquicos, intestinales y genéticos) que resulta de un desequilibrio metabólico-energético, por el cual un exceso a largo plazo de la ingesta de energía sobre el gasto conduce al almacenamiento en forma de grasa. El control del equilibrio energético lo lleva a cabo el sistema nervioso central a través de conexiones neuroendocrinas, en las que las hormonas periféricas circulantes, como la leptina y la insulina, envían señales a las neuronas especializadas del hipotálamo sobre las reservas de grasa del cuerpo e inducen respuestas apropiadas para el mantenimiento de la energía. estabilidad de estas acciones. Por lo tanto, el balance energético positivo a largo plazo, determinado por la ingesta de macronutrientes, el gasto de energía y la termogénesis, dará como resultado un aumento de peso corporal en forma de grasa, mientras que el balance energético negativo dará como resultado el efecto contrario. En esta revisión sistemática de estudios publicados entre 2008 y 2021, se discutirán los mecanismos neuroendocrinos que actúan en el control del hambre y la saciedad, y su relación con la obesidad. Demostrando así que esta regulación se lleva a cabo por un mecanismo complejo que necesita ser muy bien entendido para que la obesidad pueda ser tratada o incluso prevenida.

Palabras clave: Obesidad; Hambriento; Saciedad.

\section{Introdução}

A alimentação é uma condição básica e essencial à sobrevivência do indivíduo, sendo um processo fisiológico complexo que integra o controle do desejo de comer, o armazenamento e gasto energético. No entanto, o desajuste homeostático da ingesta alimentar e gasto energético levam a instalação de distúrbios nutricionais e fisiopatológicos como desnutrição ou obesidade (Leite \& Neto, 2009).

A obesidade é um problema de saúde pública crescente em todo o mundo. De acordo com a Organização Mundial da Saúde (2021), a obesidade tem tomado proporções epidêmicas, atingindo pessoas de todas as idades e classes sociais em todo o 
mundo. Em 2016, mais de 1,9 bilhão de adultos, com 18 anos ou mais, estavam acima do peso. Destes, mais de 650 milhões eram obesos.

É de origem multifatorial, incluindo fatores neuroendócrinos, psíquicos, intestinais e genéticos. Causa desequilíbrio metabólico-energético, predispondo ao acúmulo de tecido adiposo, o que se relaciona com diminuição da qualidade de vida, comorbidades e redução da expectativa de vida (Santanna et al., 2014). De acordo com Ferreira (2020), o ganho de peso exacerbado é fator de risco para doenças crônicas não transmissíveis, como dislipidemias, hipertensão arterial, diabetes mellitus, colecistite e síndrome de dor crônica e entre outras doenças. Além do adoecimento físico, a obesidade pode estar atrelada ao sofrimento psíquico, dado ao desajustamento emocional (alterações de humor, depressão, ansiedade, etc.) e formação de um autoconceito negativo por parte do indivíduo (Rodrigues et al., 2021).

Vários fatores atuam e interagem na regulação da ingestão de alimentos e de armazenamento de energia, contribuindo para o surgimento e a manutenção da obesidade. O controle do balanço energético é realizado pelo Sistema Nervoso Central por meio de conexões neuroendócrinas, em que hormônios periféricos circulantes, como a leptina e a insulina, sinalizam neurônios especializados do hipotálamo sobre os estoques de gordura do organismo e induzem respostas apropriadas para a manutenção da estabilidade desses estoques (Van de Sande-lee \& Velloso, 2012).

Considerando esses pressupostos, esta revisão sistemática de literatura tem como objetivo delinear os mecanismos neuroendócrinos que atuam no controle da fome e saciedade e como eles implicam na obesidade.

\section{Metodologia}

Trata-se de uma revisão sistemática da literatura, a qual agregam evidências de pesquisa para guiar a prática clínica, caracterizando-se como estratégia utilizada na investigação baseada em evidências. O percurso metodológico seguiu as seguintes etapas: definição dos objetivos e tema norteador, estabelecimento dos critérios de inclusão para seleção da amostra, seleção dos dados extraídos das pesquisas selecionadas, análise, discussão e apresentação dos resultados. Para a busca da literatura inicialmente foi feita a identificação do tema e formulada a seguinte questão norteadora: "Quais são as evidências disponíveis na literatura sobre o mecanismo de controle neuroendócrino da fome e saciedade e como ele implica na obesidade?".

A seleção dos estudos foi realizada de acordo com os seguintes critérios de inclusão: estudos que abordassem especificamente o tema norteador da pesquisa; nos idiomas português, inglês e espanhol; publicados entre 2008 e 2021; e disponíveis nas bases de dados: Literatura Latino-Americana e do Caribe em Ciências da Saúde (LILACS), Medical Literature Analysis and Retrieval System Online (MEDLINE) e Scientific Electronic Library Online (SciELO). Foram excluídos estudos que não apresentavam o resumo, que não abordavam a temática proposta ou que não respondiam à questão norteadora da pesquisa. Foram utilizados os Descritores em Ciências da Saúde (DeCS): sistema neuroendócrino, obesidade, consumo de alimentos, fome e saciedade

A busca estruturada foi realizada combinando os descritores com o operador booleano "AND", assim, foram encontrados 76 artigos, sendo que, após aplicação dos critérios de inclusão e exclusão, análise, adequação ao objetivo da revisão, observação da metodologia e didática e relevância na área científica, foram selecionados 32 artigos, e após a leitura minuciosa e crítica do conteúdo que proporcionou a organização e estruturação da revisão proposta 31 artigos foram selecionados para compor o corpus final, como consta na Figura 1. 
Figura 1- Fluxograma de triagem e seleção dos artigos.

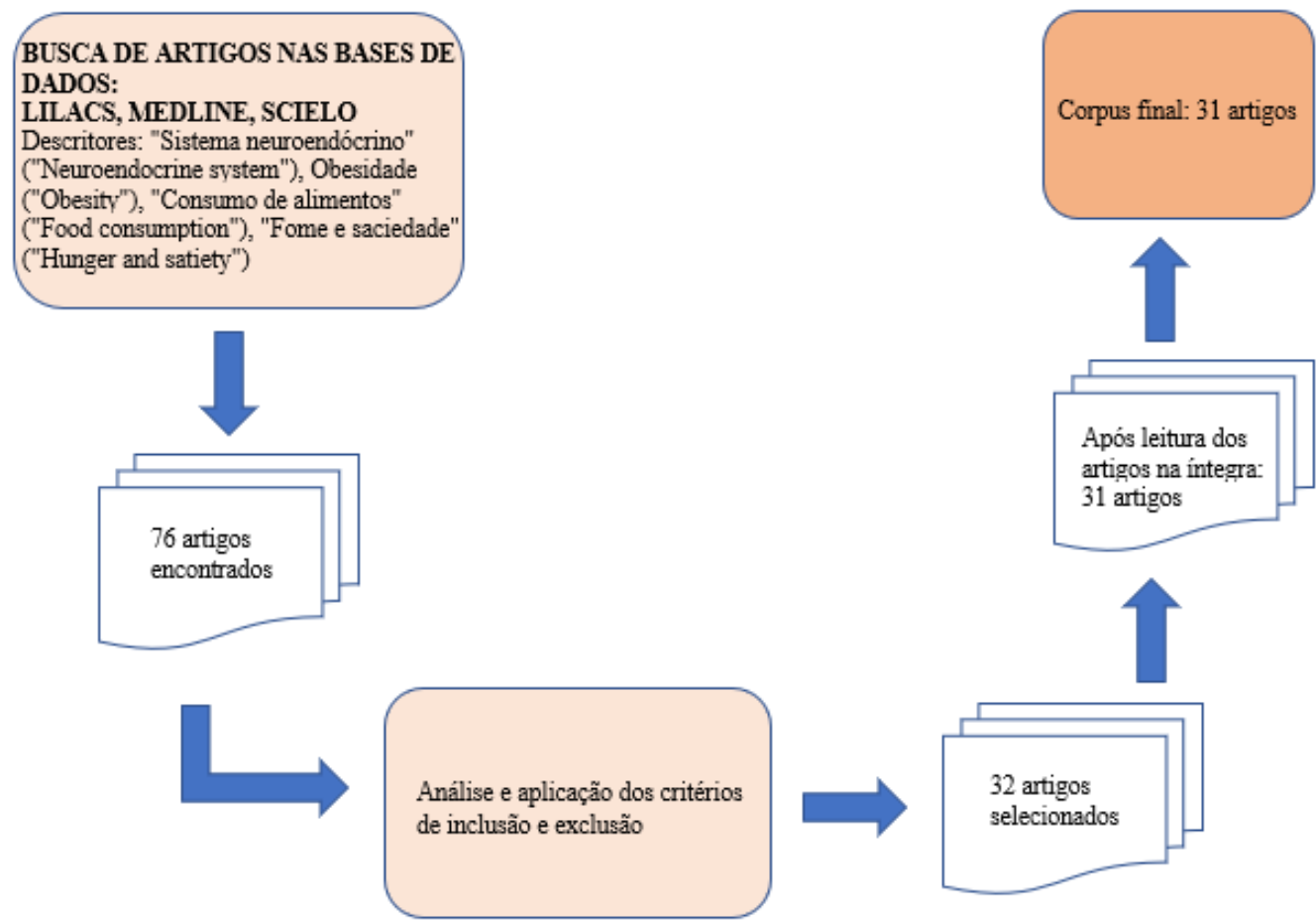

Fonte: Autores (2022).

\section{Resultados}

Para embasamento dessa revisão foram utilizados, após aplicação metodológica de adequação, 31 artigos publicados de 2008 a 2021, presentes na Tabela 1. As principais características dos estudos selecionados proporcionou distribuí-los nas seguintes áreas temáticas: 1) Obesidade e a relação neuroendócrina do balanço energético; 2) Hipotálamo e sua associação com a fome e saciedade; 3) Mecanismos neuroendócrinos da fome e saciedade; 4) Correlação neuroendócrina dos sistemas periféricos e 5) Substâncias neuroendócrinas associadas com a gênese da obesidade.

Tabela 1- Artigos que compõem a revisão.

\begin{tabular}{|l|l|l|}
\hline \multicolumn{1}{|c|}{ TíTULO } & \multicolumn{1}{|c|}{ AUTORES/ANO } & \multicolumn{1}{c|}{ REVISTA } \\
\hline Leptina e o controle neuroendócrino do peso corporal & Araújo et al., 2009 & Revista Brasileira de Medicina \\
\hline $\begin{array}{l}\text { Regulation of energy balance by a gut-brain axis and } \\
\text { involvement of the gut microbiota }\end{array}$ & Bauer et al.., 2016 & Cellular and Molecular Life Sciences \\
\hline O papel do farmacêutico na orientação da obesidade & Borsato et al., 2008 & Visão Acadêmica \\
\hline $\begin{array}{l}\text { Impacto hormonal e inflamatório de diferentes } \\
\text { composições dietéticas: ênfase em padrões } \\
\text { alimentares e fatores dietéticos específicos. }\end{array}$ & Bressan et al., 2009 & $\begin{array}{l}\text { Arquivos Brasileiros de Endocrinologia \& } \\
\text { Metabologia }\end{array}$ \\
\hline $\begin{array}{l}\text { Could there be a fine-tuning role for brain-derived } \\
\text { adipokines in the regulation of bodyweight and } \\
\text { prevention of obesity? }\end{array}$ & Brown, R. E., 2008 & McGill Journal of Medicine \\
\hline $\begin{array}{l}\text { Sinalização cerebral do apetite } \\
\text { Família: Prevenção do sobrepeso e obesidade infantil }\end{array}$ & Ferreira, A. S., 2020 & Research, Society and Development \\
\hline
\end{tabular}


Research, Society and Development, v. 11, n. 2, e33311225621, 2022

(CC BY 4.0) | ISSN 2525-3409 | DOI: http://dx.doi.org/10.33448/rsd-v11i2.25621

\begin{tabular}{|c|c|c|}
\hline $\begin{array}{l}\text { Serotonina e controle hipotalâmico da fome: uma } \\
\text { revisão }\end{array}$ & Feijó et al., 2011 & Revista da Associação Médica Brasileira \\
\hline Obesity & $\begin{array}{l}\text { González-Munieza et al., } \\
2017\end{array}$ & Nature Reviews \\
\hline Obesidad y resistencia a la leptina & $\begin{array}{l}\text { Morales Clavijo et al., } \\
2010\end{array}$ & Gaceta Médica Boliviana \\
\hline $\begin{array}{l}\text { Obese men respond to cognitive but not to catabolic } \\
\text { brain insulin signaling. }\end{array}$ & Hallschmid et al., 2008 & International Journal of Obesity \\
\hline $\begin{array}{l}\text { The hypothalamic neural-glial network and the } \\
\text { metabolic syndrome. }\end{array}$ & Jastroch et al., 2014 & $\begin{array}{l}\text { Best Practice \& Research Clinical } \\
\text { Endocrinology \& Metabolism }\end{array}$ \\
\hline $\begin{array}{l}\text { Obesidade: controle neural e hormonal do } \\
\text { comportamento alimentar }\end{array}$ & Landeiro et. al., 2011 & Revista de Ciências Médicas e Biológicas \\
\hline $\begin{array}{l}\text { Integração neuroendócrina na regulação da ingestão } \\
\text { alimentar }\end{array}$ & Leite et al., 2009 & Neurobiologia \\
\hline $\begin{array}{l}\text { Leptina e exercício físico: mecanismos para controle } \\
\text { do peso corporal. }\end{array}$ & Machado et al., 2016 & $\begin{array}{l}\text { Revista Brasileira de Prescrição e } \\
\text { Fisiologia do Exercício }\end{array}$ \\
\hline $\begin{array}{l}\text { The role of glucagon-like peptide- } 1 \text { impairment in } \\
\text { obesity and potential therapeutic implications. }\end{array}$ & Madsbad, S., 2014 & Diabetes, Obesity \& Metabolism \\
\hline $\begin{array}{l}\text { Obesity: an overview of possible role (s) of gut } \\
\text { hormones, lipid sensing and gut microbiota }\end{array}$ & Mishra et al., 2016 & Metabolism: Clinical and Experimental \\
\hline Neurobiology of food intake in health and disease & Morton et al., 2014 & Nature Reviews Neuroscience \\
\hline The role of ghrelin in reward-based eating. & $\begin{array}{l}\text { Perelló, M., \& Zigman, J. } \\
\text { M., } 2012\end{array}$ & Biological Psychiatry \\
\hline $\begin{array}{l}\text { Controle do apetite e exercício físico: uma } \\
\text { atualização }\end{array}$ & Prado et al., 2008 & Revista Brasileira de Ciência e Movimento \\
\hline $\begin{array}{l}\text { Studying the central control of food intake and } \\
\text { obesity in rats }\end{array}$ & Ribeiro, E. B., 2009 & Revista de Nutrição \\
\hline $\begin{array}{l}\text { A Escala de Analogia Visual do Apetite como } \\
\text { ferramenta de apoio na detecção do risco de fracasso } \\
\text { da persistência de perda de peso de pacientes com } \\
\text { obesidade submetidos à gastroplastia }\end{array}$ & Rodrigues et al., 2021 & Research, Society and Development \\
\hline $\begin{array}{l}\text { Inflammatory mechanisms linking obesity and } \\
\text { metabolic disease. }\end{array}$ & $\begin{array}{l}\text { Saltiel, A. R., \& Olefsky, } \\
\text { J. M. } 2017\end{array}$ & The Journal of Clinical Investigation \\
\hline $\begin{array}{l}\text { Neuromodulação Hipotalâmica: uma proposta } \\
\text { terapêutica para obesidade }\end{array}$ & Santanna et al., 2014 & Revista Medicina e Saúde de Brasília \\
\hline $\begin{array}{l}\text { Hypothalamic and brainstem neuronal circuits } \\
\text { controlling homeostatic energy balance }\end{array}$ & Schneeberger et al., 2014 & Journal of Endocrinology \\
\hline $\begin{array}{l}\text { Psychological and neural contributions to appetite } \\
\text { self-regulation }\end{array}$ & Stoeckel et al., 2017 & Obesity \\
\hline $\begin{array}{l}\text { Altered hypothalamic function in diet-induced } \\
\text { obesity. International journal of obesity }\end{array}$ & $\begin{array}{l}\text { Velloso, L. \& Schwartz, } \\
\text { M., } 2011\end{array}$ & International Journal of Obesity \\
\hline Neuroendocrine control of food intake & Valassi, et al., 2008 & $\begin{array}{l}\text { Nutrition, Metabolism and Cardiovascular } \\
\text { Diseases }\end{array}$ \\
\hline Disfunção hipotalâmica na obesidade & $\begin{array}{l}\text { Van de Sande-Lee, S., \& } \\
\text { Velloso, L. A., } 2012\end{array}$ & $\begin{array}{l}\text { Arquivos Brasileiros de Endocrinologia \& } \\
\text { Metabologia }\end{array}$ \\
\hline Obesidade: regulação hormonal & Veiga, L., 2016 & Saúde \& Tecnologia \\
\hline Obesidade: uma perspectiva plural & $\begin{array}{l}\text { Wanderley, E. N., \& } \\
\text { Ferreira, V. A., } 2010\end{array}$ & Ciência \& Saúde Coletiva \\
\hline
\end{tabular}

Fonte: Autores (2022). 


\section{Discussão}

Estudos evidenciam que o apetite é regulado por um sistema complexo, com sinais de origem central e periférica, que modula as respostas individuais do consumo e do gasto energético. Fatores neuroendócrinos vêm sendo apontados como os mais relevantes para a manutenção do balanço energético do organismo. Diante disso, atendendo os critérios de inclusão propostos metodologicamente, esta revisão sistemática da literatura identificou 31 estudos relevantes e cinco principais áreas temáticas foram identificadas e explanadas:

\section{Obesidade e a relação neuroendócrina do balanço energético}

Na obesidade, a genética contribui com alguns casos e outros fatores, como comportamento alimentar, sedentarismo e prática da atividade física possuem maior influência sobre o excesso de gordura corporal (Borsato et al., 2008). Segundo Landeiro \& Quarantini (2011) evidências sugerem que a ingestão de nutrientes, assim como o metabolismo destes nutrientes, podem ser controlados ou modulados por estruturas neurais e por sistemas neuroquímicos e neuroendócrinos específicos. Alterações nestes sistemas neurais podem estar associadas às mudanças no comportamento alimentar. Pesquisas sobre o controle neuroendócrino do metabolismo energético identificaram os peptídeos leptina e insulina como os dois maiores sinalizadores de adiposidade que informam ao cérebro a quantidade de energia corporal armazenada como excessiva (Hallschmid et al., 2008).

Fatores neuroendócrinos vêm sendo apontados como os mais relevantes para a manutenção do balanço energético do organismo, evitando a perda ou ganho de peso. Diversos sinais, pela interação com neurônios do núcleo arqueado no terceiro ventrículo hipotalâmico, são capazes de aumentar a termogênese e estimular a saciedade em momentos de muita oferta energética. Esses sinais são capazes também de promover o contrário, estimulando a fome e inibindo a termogênese em momentos de privação (Landeiro \& Quarantini, 2011).

\section{Hipotálamo e sua associação com a fome e saciedade}

Atualmente, busca-se compreender a relevância fisiológica desses sistemas de controle e o papel que desempenham na obesidade. Esse complexo circuito age de forma integrada, interferindo nas sensações de fome, saciedade, esvaziamento e plenitude gástrica (Ribeiro, 2009).

Estudos de lesões hipotalâmicas realizadas entre o final da década de 1930 e o início da década de 1960, ofereceram a primeira evidência de um papel do cérebro no controle do peso corporal e na patogênese da obesidade e diabetes. Com base nesses e em estudos subsequentes, os núcleos hipotalâmicos laterais e ventromediais foram identificados como áreas cerebrais potencialmente importantes que controlam a ingestão de alimentos e o gasto de energia (Velloso \& Schwartz, 2011). O controle hipotalâmico do apetite é um mecanismo complexo e está ligado não somente ao cérebro, mas a sistemas e sinais periféricos que atuam via circuitos de recompensa (Landeiro \& Quarantini, 2011).

A manipulação de áreas específicas do hipotálamo pode alterar de maneira confiável o comportamento alimentar, o metabolismo energético e a massa corporal, duas teorias importantes surgiram na década de 1950 para explicar a fisiologia desse sistema. A energia armazenada no tecido adiposo é comunicada ao hipotálamo através de sinais circulantes que servem para ajustar a ingestão de alimentos em resposta à variação no tamanho do depósito de energia. Por outro lado, foi proposto também que a variação nas concentrações de glicose no plasma ou no tecido é um sinal fundamental que informa o sistema nervoso central sobre a disponibilidade de energia. Essas duas hipóteses foram posteriormente denominadas modelos adipostático e glucostático de homeostase energética (Velloso \& Schwartz, 2011). 


\section{Mecanismos neuroendócrinos da fome e saciedade}

As áreas cerebrais que são responsáveis pela homeostase energética recebem sinais neuronais e hormonais de acordo com as necessidades do indivíduo, disposição de energia e metabólitos (González-Munieza et al., 2017). Nesse mecanismo estão relacionados sinais aferentes de curta ou longa duração que são repassados ao cérebro através do nervo vago ou pela via sistêmica e envolvendo também hormônios provenientes do trato gastrointestinal e tecido adiposo de acordo disponibilidade energética do indivíduo. Para que haja respostas a esses estímulos, as vias eferentes são responsáveis por transmitir impulsos de ativação ou inibição de neurônios orexígenos (responsáveis pela sensação de fome) e anorexígenos (responsáveis pela saciedade) localizados em diversas áreas do hipotálamo (Veiga, 2016).

Para Wanderley \& Ferreira (2010), a regulação do peso corporal é controlada por meio de hormônios e neuropeptídeos, que estão sob o comando de núcleos hipotalâmicos. No entanto, o princípio da alimentação não parece ser regulado principalmente por sinais bioquímicos (Brown, 2008; Valassi et al., 2008). O início da refeição parece ser estimulado bioquimicamente somente em casos severos de privação energética, enquanto que normalmente é controlado por fatores sociais, culturais e ambientais, que estão estreitamente ligados ao estilo de vida do indivíduo (Brown, 2008).

De acordo com a "teoria glicostática", o "centro da fome” está localizado na área lateral hipotalâmica, onde a percepção de baixa glicose sanguínea é reconhecida, e assim, consequentemente é estimulado o consumo alimentar. Após a refeição, o "centro da saciedade", localizado na região ventromedial do hipotálamo, é ativado desencadeando a inibição do "centro da fome", cessando a alimentação (Valassi et al., 2008).

Mietussnyder \& Lustig (2008) apresentaram um modelo teórico que integra os diferentes fatores cerebrais envolvidos na alimentação - saciação, recompensa e estresse - para explicar os sistemas que provocam os mecanismos fisiológicos de sobrevivência humana que se sobrepõem à homeostase e contribuem para o ganho de peso. Para os autores, os centros de controle que regulam o apetite e o gasto de energia situam-se em regiões profundas, entre três áreas do sistema límbico primitivo compreendendo hipotálamo, área tegmental ventral e amígdala. Cada uma destas áreas percebe uma sensação distinta mas complementar, que leva ao comportamento alimentar.

Projeções descendentes dos neurônios hipotalâmicos podem modular a ingestão de alimento modificando a capacidade de controle da saciedade. Além disso, a ação crônica da insulina na área tegmental ventral desregula as vias hedônicas da recompensa que, por seu turno, aumentam o comportamento de busca do alimento, especialmente os ricos em gordura ou açúcar, e isto resulta na ingestão excessiva de energia. E, finalmente, a ativação crônica da amígdala, sob condições de estresse, depressão ou ansiedade, aumenta a secreção de cortisol. Esta produção excessiva de cortisol leva ao acúmulo de gordura visceral e aumento na sinalização da lecitina, perpetuando o ciclo vicioso de ganho de peso (Mietussnyder \& Lustig, 2008).

\section{Correlação neuroendócrina dos sistemas periféricos}

As interações do sistema neuroendócrino juntamente com o trato gastrointestinal e com o tecido adiposo são expressos através de sinais fisiológicos de forma a regular o apetite, por meio de mecanismos de comunicação expressos no hipotálamo e no tronco encefálico. Estas regiões neurais são constituídas por neurônios heterogêneos com particularidades neuroquímicas e biofísicas, os quais possuem receptores específicos para leptina, grelina, GLP-1 e CCK (Schneeberger et al., 2014).

Mediada por mecanorreceptores e quimiorreceptores periféricos, a via de sinalização vagal é a responsável pela interação neural, que na presença ou ausência de alimento no trato gastrointestinal ativam o sistema neuroendócrino de curto prazo e secretam mais de 20 peptídeos hormonais de ação orexígena e anorexígena, os quais são encarregados de controlar a 
fome e da saciedade. Conforme oscilações das reservas energéticas de longo prazo, tecido adiposo também secreta hormônios, sendo a leptina o principal hormônio adipositário (Damiani \& Damiani, 2011).

$\mathrm{O}$ tecido adiposo expressa e secreta uma variedade de peptídeos bioativos conhecidos como adipocinas (substâncias orexígenas e anorexígenas) que possuem ação tanto autócrina quanto parácrina além do nível sistêmico (endócrina) e desenvolvem um importante papel no controle da homeostase energética, estando diretamente relacionado com a obesidade (Valassi et al., 2008).

Segundo Mishra et al. (2016), a adipocina leptina, é o peptídeo de maior influência na homeostasia energética. Os níveis circulantes de leptina são proporcionais ao estado nutricional e às reservas de tecido adiposo, encontrando-se em concentrações mais elevadas no tecido adiposo subcutâneo quando comparado com o tecido adiposo visceral. Em condições fisiológicas normais, a leptina é um potente inibidor de estímulos neuronais orexígenos (Damiani \& Damiani, 2011). Consoante a isso, Araújo et al. (2009) afirma que aumento de concentração sérica de leptina está relacionado com o aumento de tecido adiposo, o que pode estar envolvido tanto com o número de células adiposas quanto a indução da transcrição do gene ob. (responsável pela leptina).

\section{Substâncias neuroendócrinas associadas com a gênese da obesidade}

O papel primário da leptina é sinalizar o SNC sobre os estoques de energia no tecido adiposo, de forma que o cérebro promova respostas metabólicas que permitam os ajustes necessários para equilibrar o gasto e o consumo energético. Os estímulos de longo prazo estão associados às oscilações entre a lipólise e a lipogênese, decorrente de períodos de jejum prolongado ou antagonicamente ao acúmulo de massa gorda (Machado et al., 2015).

A leptina atravessa a barreira hematoencefálica e se liga ao seu receptor (ObR ou LepR), localizado no hipotálamo. Os neurônios que expressam os receptores estão localizados principalmente em dois núcleos hipotalâmicos: o ventromedial, com funções anorexígenas e pró-termogênicas, e o hipotálamo lateral, com funções orexígenas e antitermogênicas (Santanna et al., 2014).

A ação primária da leptina no controle do balanço energético ocorre em dois tipos de neurônios no núcleo hipotalâmico arqueado (Valassi et al., 2008): 1 - Neurônio que expressam pró-opiomelanocortina (POMC): são precursores do hormônio estimulante dos melanócitos ( $\alpha-\mathrm{MSH}) ; 2$ - Neurônios que expressam a cocaína e a anfetamina, pela regulação da enzima transcriptase (CART): substâncias consideradas anorexígenas, ou seja, ligadas aos mecanismos de inibição da ingestão alimentar e aumento do gasto energético total, via ativação do sistema nervoso simpático.

Ademais, na regulação do balanço energético a leptina atua em conjunto com a colecistoquinina (CCK), que após a ingestão alimentar é liberada no intestino e age no nervo vagal aferente para ativar neurônios do núcleo hipotalâmico ventromedial que estimula a redução do consumo de alimentos (Santanna et al., 2014).

A insulina, também tem um papel significativo na fisiologia da obesidade, visto que é produzida pelas células $\beta$ do pâncreas cuja a secreção basal e a secreção estimulada desse hormônio pela glicose são diretamente proporcionais à adiposidade (Van de Sande-lee \& Velloso, 2012). A insulina possui ações neurais no controle do balanço energético semelhantes às da leptina, em contraste com seus efeitos anabólicos sobre tecidos periféricos de regulação da glicose, sua ação hipotalâmica produz efeitos catabólicos (Santanna et al. 2014).

A atividade da leptina no hipotálamo é modulada positivamente pela insulina e vice-versa. Uma queda na gordura corporal é sentida no núcleo arqueado do hipotálamo como uma diminuição da concentração dos níveis de insulina e leptina. Isso causa supressão nos sinais anorexígenos e estímulo nos sinais orexígenos, o que gera aumento da ingesta alimentar, queda no gasto energético e depósito de massa gorda. De forma contrária, em momentos de ingestão alimentar, ocorre aumento da sensibilidade a leptina e insulina, com sensação de saciedade. Portanto, a grande maioria dos indivíduos obesos não apresenta 
Research, Society and Development, v. 11, n. 2, e33311225621, 2022

(CC BY 4.0) | ISSN 2525-3409 | DOI: http://dx.doi.org/10.33448/rsd-v11i2.25621

deficiência de leptina ou insulina, mas resistência à ação central dos sinais de adiposidade (Santanna et al., 2014). A função da leptina no balaço energético, tanto pela inibição da ingestão quanto pelo aumento do gasto energético, tem a importante função de manutenção da massa corporal e prevenção da obesidade (Brown, 2008).

Os níveis circulantes de leptina correlacionam-se diretamente com as reservas de tecido adiposo, ou seja, o aumento da adiposidade induz ao aumento da produção de leptina, enquanto uma diminuição de massa gorda está associada a uma redução da sua expressão. Estudos sugerem que o perfil da dieta de indivíduos obesos acarreta em níveis circulantes de leptina muito elevados que são capazes de saturar os receptores desse hormônio e alterar a permeabilidade hematoencefálica, configurando uma falha no sistema de comunicação entre o tecido adiposo e o SNC. A insensibilidade dos receptores hipotalâmicos caracteriza o estado de resistência à leptina e devido a esta condição indivíduos obesos apresentam hiperfagia e compulsão alimentar (Clavijo \& Garces, 2010).

A grelina é um estimulador de apetite em nível de hipotálamo que trabalha em curta escala de tempo entre refeições (Prado et al., 2008). De acordo com Perelló \& Zigman (2012), ainda que os níveis de grelina estejam diminuídos imediatamente após a ingestão alimentar, indivíduos obesos apresentam tendências à hiperfagia, mesmo em estado alimentado. Este comportamento pode ser explicado pela estimulação sensorial que amplia o valor de recompensa dos alimentos e promove o comportamento hedônico, baseado no consumo excessivo de refeições palatáveis e densas em energia.

Em ação inversa à grelina, hormônios anorexígenos como a CCK e o GPL-1, atuam na sinalização neuroendócrina de curto prazo. A secreção intestinal desses hormônios no período pós-absortivo estabelece um sistema de comunicação bidirecional denominado eixo cérebro-intestino. Este eixo é vital para a manutenção do equilíbrio energético, pois os sinais originados no intestino não só reduzem o consumo alimentar, como também demonstram controlar o gasto de energia (Bauer et al., 2015).

O pró-ópio-melanocortina (POMC) é expresso no núcleo arqueado e precursor da $\alpha$-Melanotropina ( $\alpha \mathrm{MSH})$, que representa um dos reguladores mais importantes do balanço energético (mediado pelos receptores MC3R e MC4R) (Valassi et al., 2008).

Os fatores intestinais têm participação importante na fisiopatologia da obesidade. A absorção, ou mesmo a presença de alimento no trato gastrintestinal, contribui para a modulação do apetite e para a regulação de energia (Santanna et al., 2014). A CCK é secretada no intestino delgado proximal em resposta à ingestão de ácidos graxos e aminoácidos (Mishra et al., 2016). A secreção de CCK é diretamente proporcional ao conteúdo lipídico e proteico da refeição e sua ação estimula a contração da vesícula biliar para a liberação de sais biliares no duodeno. Em seu papel na regulação do apetite a CCK atua como inibidor do esvaziamento gástrico e da motilidade gastrointestinal, favorecendo a absorção de nutrientes. A CCK também provoca o aumento da distensão abdominal promovendo a sensação de plenitude e, consequentemente, a diminuição da ingestão alimentar (Veiga, 2016).

Conforme Bressan et al. (2009), a secreção de CCK é estimulada pela entrada e assimilação de ácidos graxos nos enterócitos. Porém, as gorduras saturadas inibem a síntese e liberação de neuropeptídeos anorexígenos. O GLP-1 também é um peptídeo intestinal pertencente a classe dos hormônios anorexígenos, sendo a sua liberação afetada pelos macronutrientes presentes no lúmen intestinal, principalmente os carboidratos. A concentração de GLP-1 circulante é baixa durante o período de jejum e sofre um ligeiro aumento após refeição, uma vez que é degradada quase que imediatamente pela enzima dipeptidil peptidase-4. A principal função do GLP-1 é a regulação do nível de glicose no sangue. A liberação desse hormônio aumenta a sensibilidade à insulina e inibe a ação do glucagon, agindo como uma potente incretina. O efeito insulinotrópico do GLP-1 retarda o esvaziamento gástrico e reduz a motilidade gástrica, contribuindo para a diminuição do apetite (Mishra et al., 2016).

De acordo com Santanna et al. (2014), outro inibidor da ingestão alimentar é o peptídeo YY ou PYY, constituído de 34 aminoácidos e secretado por células presentes no revestimento mucoso do cólon e do intestino delgado. Sua secreção ocorre 
com a entrada do bolo alimentar e, portanto, os níveis plasmáticos deste hormônio aumentam quase imediatamente após a ingestão de alimentos. Ele age inibindo a liberação de NPY por neurônios orexígenos através do receptor PYY. Dado que o NPY é responsável pela sensação de fome e o PYY a inibe, este último, juntamente com o GLP-1, são importantes para o controle da ingestão alimentar, pois são responsáveis pela sensação de saciedade. Indivíduos obesos apresentam menor elevação dos níveis de PYY pós-prandial, especialmente em refeições noturnas, o que leva a uma ingestão calórica maior.

Determinações de serotonina no hipotálamo revelaram que suas concentrações são baixas durante períodos de jejum, aumentam em antecipação à chegada de alimento e apresentam um pico durante a refeição, especialmente em resposta a carboidratos. A serotonina é produzida a partir do aminoácido triptofano, presente na dieta. As concentrações de triptofano no sangue variam de acordo com a quantidade de carboidratos ingerida. Assim, o aumento do triptofano no sangue com o consequente aumento da serotonina no encéfalo é uma provável explicação para a ocorrência de bom humor após a ingestão de alimentos específicos. Assim sendo, o prazer alimentar pode produzir comportamentos viciosos e compulsivos, levando à obesidade (Santanna et al., 2014). De acordo com Feijó et al. (2011), na presença de níveis normais de serotonina, o indivíduo se encontra mais facilmente saciável e tem maior controle na ingestão de doces.

Diante da relevância da ação endócrina sob o controle da ingestão alimentar e do gasto energético, é necessário salientar que a homeostase energética parece prejudicada em indivíduos obesos não só pela resistência hormonal, mas também pela inflamação sistêmica de baixo grau originada pela obesidade, impedindo a correta ação hormonal que acarreta em maior ganho de peso e menor gasto de energia (Morton et al., 2014).

A inflamação associada à obesidade tem sido relacionada com alterações na função hipotalâmica, particularmente em áreas que regulam a homeostase energética e o metabolismo sistêmico (Saltiel \& Olefsky, 2017). A inflamação hipotalâmica induzida pela dieta afeta a expressão dos neurônios envolvidos no eixo cérebro-intestino, comprometendo a capacidade de assimilação de nutrientes e de sinalização de hormônios como a grelina, CCK, GLP-1 e leptina, principais gatilhos neuroendócrinos reguladores do comportamento alimentar e o gasto energético (Jastroch et al., 2014).

Desta forma, embora as concentrações hormonais possam estar em níveis normais em indivíduos obesos, o estado de inflamação reduz a expressão dos receptores e promovem resistência hormonal, impossibilitando a autorregulação do apetite (Stoeckel et al., 2017).

\section{Conclusão}

Infere-se, através dos estudos revisados, que a regulação da fome e saciedade implica por conseguinte nas alterações da massa corporal. Essa regulação é realizada por um mecanismo complexo, que precisa ser compreendido e utilizado em diagnósticos e intervenção nos distúrbios de ingestão alimentar. Levando-se em consideração esta multicausalidade, é indispensável agregar conhecimentos sobre o controle neuroendócrino da fome e saciedade na regulação do balanço energético, como também conhecer os parâmetros bioquímicos e moleculares envolvidos de forma a auxiliar na definição de estratégias terapêuticas e de prevenção de doenças como a obesidade. Dessa forma, faz-se necessário o desenvolvimento de futuros trabalhos de maneira a contribuir de maneira significativa à comunidade científica, visto que há uma carência de estudos sobre a temática abordada.

\section{Referências}

Araújo, T. F. D., Guimarães, D. F., Ferreira, F. R., Luz, J. C. D. M., \& Spini, V. B. M. G. (2009). Leptina e o controle neuroendócrino do peso corporal. RBM Revista Brasileira de Medicina, 66(10), 325-30.

Bauer, P. V., Hamr, S. C., \& Duca, F. A. (2016). Regulation of energy balance by a gut-brain axis and involvement of the gut microbiota. Cellular and molecular life sciences, 73(4), 737-755. https://doi.org/10.1007/s00018-015-2083-z 
Research, Society and Development, v. 11, n. 2, e33311225621, 2022

Borsato, D., Zanetti, C., Kalegari, M., Zanin, S., \& Miguel, M. (2008). O papel do farmacêutico na orientação da obesidade. Visão acadêmica, 9(1). http://dx.doi.org/10.5380/acd.v9i1.14636

Bressan, J., Hermsdorff, H. H., Zulet, M. Á., \& Martínez, J. A. (2009). Impacto hormonal e inflamatório de diferentes composições dietéticas: ênfase em padrões alimentares e fatores dietéticos específicos. Arquivos Brasileiros de Endocrinologia \& Metabologia, 53, 572-581. https://doi.org/10.1590/S000427302009000500010

Brown, R. E. (2008). Could there be a fine-tuning role for brain-derived adipokines in the regulation of bodyweight and prevention of obesity?.McGill Journal of Medicine: MJM, 11(2), 177-184

Damiani, D., \& Damiani, D. (2011). Sinalização cerebral do apetite. Revista da Sociedade Brasileira de Clínica Médica, 9(2), 138-45.

Feijó, F. D. M., Bertoluci, M. C., \& Reis, C. (2011). Serotonina e controle hipotalâmico da fome: uma revisão. Revista da Associação Médica Brasileira, 57, 74-77.

Ferreira, A. de S. (2020). Consulta de enfermagem na Estratégia Saúde da Família: Prevenção do sobrepeso e obesidade infantil. Research, Society and Development, 9(5), e17952610. https://doi.org/10.33448/rsd-v9i5.2610

González-munieza, P., Mártinez-gonzález, M.A., Hu, F.B., Després, J.P., Matsuzawa, Y., Loos, R.J.F., Moreno, L.A., Bray, G.A., \& Martinez, J.A. (2017). Obesity. Nature reviews. Disease primers, 3, 17034. https://doi.org/10.1038/nrdp.2017.34

Hallschmid, M., Benedict, C., Schultes, B., Born, J., \& Kern, W. (2008). Obese men respond to cognitive but not to catabolic brain insulin signaling. International journal of obesity, 32(2), 275-282. https://doi.org/10.1038/sj.ijo.0803722

Jastroch, M., Morin, S., Tschöp, M. H., \& Yi, C. X. (2014). The hypothalamic neural-glial network and the metabolic syndrome. Best Practice \& Research Clinical Endocrinology \& Metabolism, 28(5), 661-671. https://doi.org/10.1016/j.beem.2014.02.002

Landeiro, F. M., \& Quarantini, L. de C. (2011). Obesidade: Controle Neural e Hormonal do Comportamento Alimentar. Revista de Ciências Médicas e Biológicas, 10(3), 236-245. https://doi.org/10.9771/cmbio.v10i3.5883

Leite, L. D., \& Neto, J. (2009). Integração neuroendócrina na regulação da ingestão alimentar. Neurobiologia, 72(2), $127-143$.

Machado, W., Monteiro, E. R., \& Salerno, V. (2016). Leptina e exercício físico: mecanismos para controle do peso corporal. Revista Brasileira de Prescrição e Fisiologia do Exercício, 9(54), 471-480.

Madsbad S. (2014). The role of glucagon-like peptide-1 impairment in obesity and potential therapeutic implications. Diabetes, Obesity \& Metabolism, 16(1), 9-21. https://doi.org/10.1111/dom.12119

Mishra, A. K., Dubey, V., \& Ghosh, A. R. (2016). Obesity: An overview of possible role(s) of gut hormones, lipid sensing and gut microbiota. Metabolism: clinical and experimental, 65(1), 48-65. https://doi.org/10.1016/j.metabol.2015.10.008

Morales Clavijo, M., \& Carvajal Garcés, C. F. (2010). Obesidad y resistencia a la leptina. Gaceta médica boliviana, 33(1), 63-68.

Morton, G. J., Meek, T. H., \& Schwartz, M. W. (2014). Neurobiology of food intake in health and disease. Nature reviews. Neuroscience, 15(6), 367-378. https://doi.org/10.1038/nrn3745

Perelló, M., \& Zigman, J. M. (2012). The role of ghrelin in reward-based eating. Biological psychiatry, 72(5), 347-353. https://doi.org/10.1016/j.biopsych.2012.02.016

Prado, E. S., Almeida, R. D. D., \& Melo, L. A. D. (2008). Controle do apetite e exercício físico: uma atualização. Revista Brasileira de Ciência e Movimento, 16(2): 109-116

Ribeiro, E. B. (2009). Studying the central control of food intake and obesity in rats. Revista de Nutrição, 22 (1), 163-171. https://doi.org/10.1590/S141552732009000100015

Rodrigues, G. B. de O., Lima, G. P., Sampaio, H. A. de C., Pinto, F. J. M., Carneiro, C., Cordeiro, M. D., \& Carioca, A. A. F. (2021). A Escala de Analogia Visual do Apetite como ferramenta de apoio na detecção do risco de fracasso da persistência de perda de peso de pacientes com obesidade submetidos à gastroplastia. Research, Society and Development, 10(7), e50410716568. https://doi.org/10.33448/rsd-v10i7.16568

Saltiel, A. R., \& Olefsky, J. M. (2017). Inflammatory mechanisms linking obesity and metabolic disease. The Journal of Clinical Investigation, 127(1), 1-4. https://doi.org/10.1172/JCI92035

Santanna, S., Caiado, N. M., \& Silva, L. D. (2014). Neuromodulação Hipotalâmica: uma proposta terapêutica para obesidade. Revista Medicina e Saúde de Brasília, 3(2), 192-204.

Schneeberger, M., Gomis, R., \& Claret, M. (2014). Hypothalamic and brainstem neuronal circuits controlling homeostatic energy balance. The Journal Of Endocrinology, 220(2), T25-T46. https://doi.org/10.1530/JOE-13-0398

Stoeckel, L. E., Birch, L. L., Heatherton, T., Mann, T., Hunter, C., Czajkowski, S., Onken, L., Berger, P. K., \& Savage, C. R. (2017). Psychological and neural contributions to appetite self-regulation. Obesity (Silver Spring, Md.), 25 Suppl 1(Suppl 1), S17-S25. https://doi.org/10.1002/oby.21789

Valassi, E., Scacchi, M., \& Cavagnini, F. (2008). Neuroendocrine control of food intake. Nutrition, metabolism, and cardiovascular diseases. NMCD, 18(2), 158-168. https://doi.org/10.1016/j.numecd.2007.06.004 
Research, Society and Development, v. 11, n. 2, e33311225621, 2022

(CC BY 4.0) | ISSN 2525-3409 | DOI: http://dx.doi.org/10.33448/rsd-v11i2.25621

Van de Sande-Lee, S., \& Velloso, L. A. (2012). Disfunção hipotalâmica na obesidade. Arquivos Brasileiros de Endocrinologia \& Metabologia, 56 (6), 341 350. https://doi.org/10.1590/S0004-27302012000600001

Veiga, L. (2016). Obesidade: regulação hormonal. Saúde \& Tecnologia, (16), 5-15. http://dx.doi.org/10.25758/set.1421

Velloso, L. A., \& Schwartz, M. W. (2011). Altered hypothalamic function in diet-induced obesity. International Journal Of Obesity, 35(12), 1455-1465. https://doi.org/10.1038/ijo.2011.56

Wanderley, E. N., \& Ferreira, V. A. (2010). Obesidade: uma perspectiva plural. Ciência \& Saúde Coletiva, 15(1), 185-194. https://doi.org/10.1590/S141381232010000100024

World Healthy Organization (WHO). (2021). Obesity and overweight. Genebra. https://www.who.int/news-room/fact-sheets/detail/obesity-and-overweight 